\title{
Mobile microscopy for the examination of blood samples
}

\author{
Juliane Pfeil ${ }^{1}$, Marcus Frohme ${ }^{1 凶}$, Katja Schulze ${ }^{2}$ \\ 1 Division Molecular Biotechnology and Functional Genomics, Technical University of Applied Sciences, Wildau, Germany \\ ${ }^{2} \mathrm{CSO}$ and Computer Vision Expert, Oculyze GmbH, Wildau, Germany
}

Competing interests: JP none; MF none; KS The submitted work was performed in cooperation with the company Oculyze GmbH, Wildau Germany. Oculyze works in the field of mobile microscopy and computer vision. Therefore, Oculyze has a vested interest in the success of this field.

\section{Abstract}

The analysis of blood is one of the best possibilities to diagnose and control diseases and deficiency symptoms. Common blood tests that are performed in medical laboratories are time-consuming and work-intensive. In under-developed areas, there is often also a lack of specialised staff and facilities. The development of a mobile microscopic system that contains an automated image analysis and that can be used via a smartphone, could represent a valuable help to improve the diagnostic care, especially in those areas. It aims to enable a very fast, cheap, location- and knowledge-independent application for many use cases.

\section{Introduction}

Microscopy was already developed in the late 16th century and was initially more for wealthy peoples leisure than science. In modern days, it is still one of the most important tools in diagnostics and process monitoring. In most cases, a microscopic analysis requires expensive devices and trained specialists. In addition, investigations can often only be carried out in laboratories and they are very complex. The documentation is done via computerassisted camera-systems, but automatic image analysis is rarely present.

As "Molecular Biotechnology and Functional Genomics" research group on automated microscopy and image analysis, we developed an automated recognition system of phytoplankton species for the evaluation of freshwaters trophic levels (Schulze et al., 2013). The requirement of a mobile and affordable microscopic system that enables image analysis "in the field" was an obvious observation from this project.

Based on these results the company Oculyze ${ }^{1}$ was founded with the objective to develop such a system and enable everyone to be a microscopy specialist with the support of machine learning analysis algorithms. The company reached that goal by providing a mobile microscopy solution and custom image recognition software for different biological samples.

The recently developed smartphone microscope (Figure 1) was used as a technical and economical "proof of concept" for the analysis of yeast in beer breweries.

${ }^{1}$ http://www.oculyze.de

\section{Article history}

Received: 16 November 2017

Accepted: 17 December 2018

Published: 05 February 2018
With this system, the control of concentration and viability of yeast cells is possible which is helpful to safeguard good and maintain a constant quality of beer (White and Zainasheff, 2010).

For the analysis of blood, there are several microscopic approaches commonly used as diagnostic tools. A specialist can count red and white blood cells, differentiate cell groups, investigate morphological changes and identify or quantify parasites etc. For

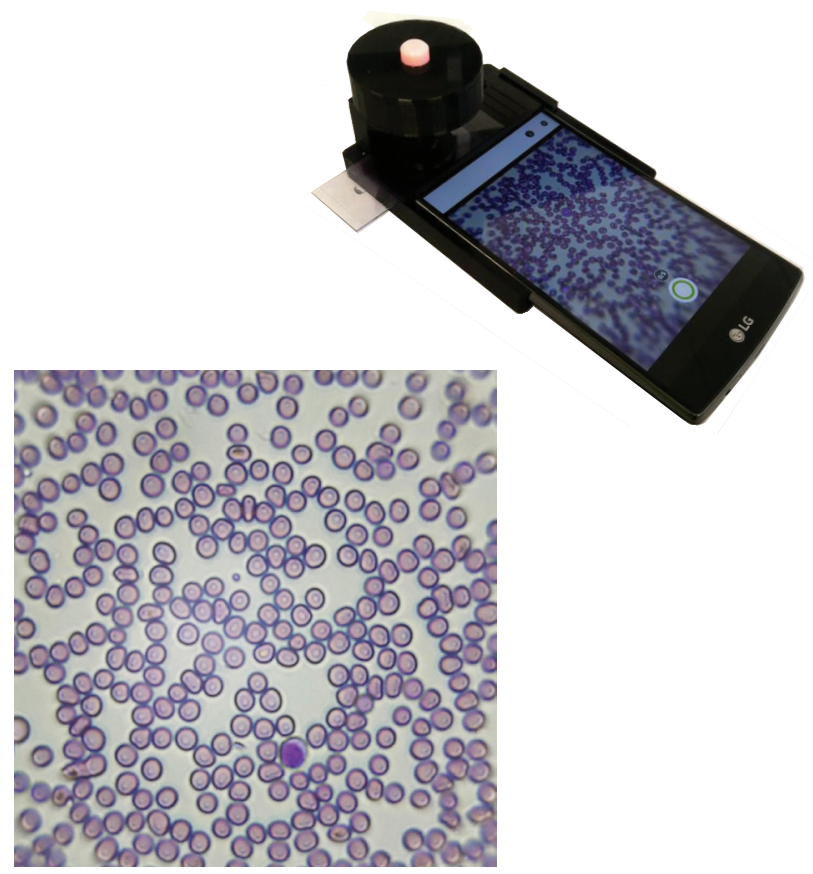

Figure 1. Recent Oculyze smartphone microscope. A stained blood sample is shown with a magnification of 400x. The used Hemacolor ${ }^{\circ}$ chemicals are staining red blood cells bright purple and white blood cells dark purple.

(c) 2018 Pfeil et al.; the authors have retained copyright and granted the Journal right of first publication; the work has been simultaneously released under a Creative Commons Attribution Licence, which allows others to share the work, while acknowledging the original authorship and initial publication in this Journal. The full licence notice is available at http://journal.embnet.org. 
example, lower levels of red blood cell counts are an indicator of anaemia. In most cases, this disease is easy to cure because it is mainly caused by iron deficiency. Unfortunately, it is an under-diagnosed illness because of the elaborate application of a microscopic analysis (WHO, 2008). A fast and reliable test could improve the welfare of a large percentage of the population.

There are also many less developed countries in tropical regions that have an endemic problem of malaria infections. Still, so far, the gold standard of diagnosis is the microscopic validation of these parasites. The lack of an adequate infrastructure (laboratories, specialised employees) hinders a fast and reliable diagnosis and treatment (Malaria Atlas Project, 2017).

A mobile microscopic system can be used as a point-of-care diagnostic (POCD) tool directly at the patient bed or in remote areas with poor medical supply.

\section{Materials, Methodologies Techniques}

Oculyze has developed a mobile microscopy system that can be used without expert knowledge (Figure 1). A combination of an optical module, a corresponding smartphone and automated image analysis, enables different cell counting approaches. The whole system (containing hard- and software) is cheaper than a cell counter and does not require trained personnel compared to analysing samples with a laboratory microscope. The optical module enables a 400x magnification with a high resolution of samples. With the connected smartphone, the user can record and analyse microscopic images automatically. Documentation is stored in the cloud, readily available from any internet connected device. The software currently comprises an Android application and cloud-based image analysis algorithms. The captured images are transmitted via mobile network to the cloud server and the results are displayed on the screen after a few seconds.

An uncomplicated adaption of the image analysis software to novel scientific issues is possible. For more challenging tasks, e.g. blood analysis, further developments for an advanced mobile microscope catching the following requirements are necessary (Figure 2):

- development of a mobile microscope that can be used with any smartphone or mobile device;

- automatic image recording and adjustable sample holder;

- fast and reliable image analysis for blood samples based on neural networks and deep learning.

\section{Results}

At first, requirements for a blood analysis system were evaluated. There are several indicators for the objective to develop a POCD tool:

- simple and fast sample preparation;

- easy to use;

- highly accurate results for diagnostics.

Herein, the sample handling should preferably be carried out by the patient itself - also to avoid the risk of infections. A drop of blood from the fingertip is enough to investigate diagnostic target parameters. It is placed on a microscope slide and white blood cells, thrombocytes and possibly parasites are stained with a DNA-sensitive dye. Operability by user-friendly application needs to be achieved. Yet, the most important point is the diagnostic reliability, which will be achieved with the development of deep learning algorithms and neural networks. The accuracy of this approach is very promising because the results of artificial intelligence programs are the superior
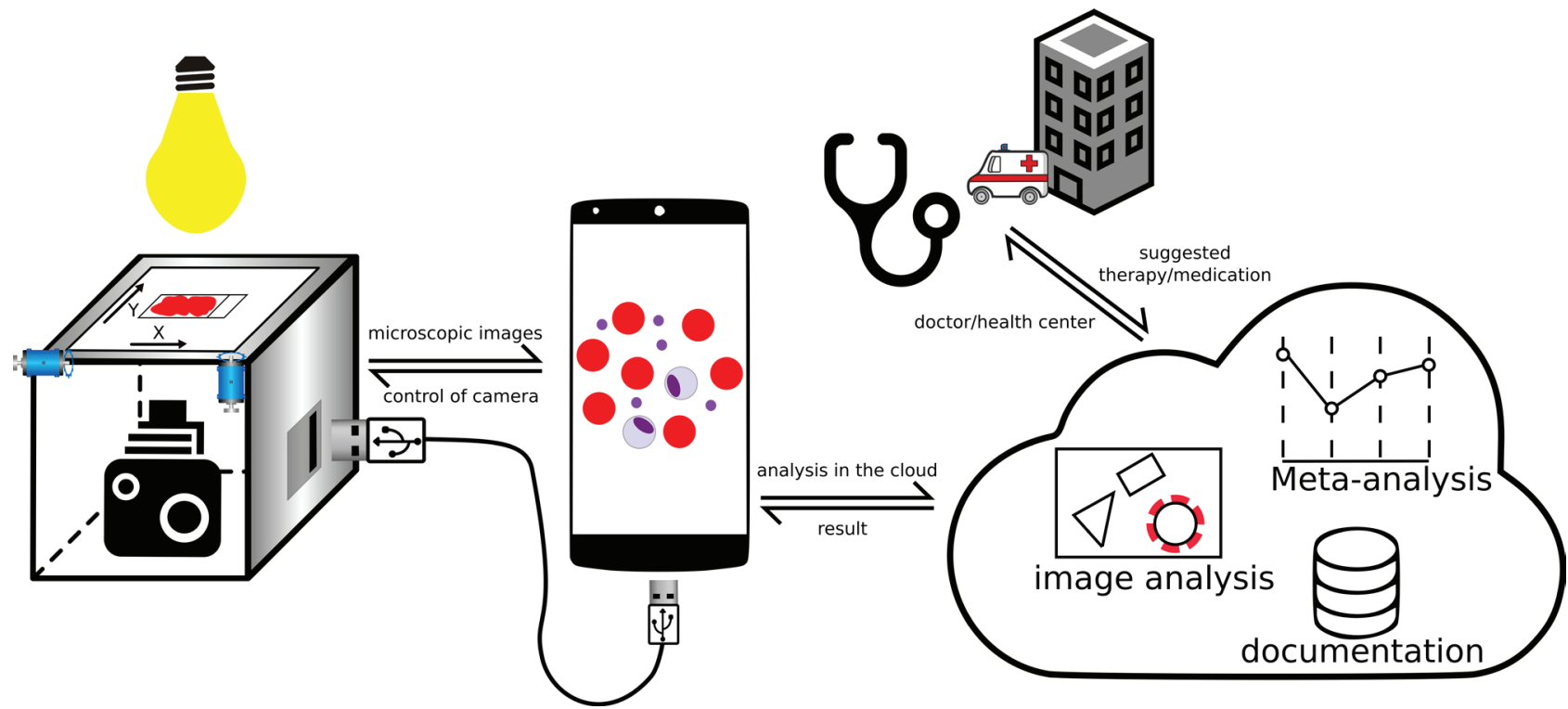

Figure 2. The planned, mobile microscope, consisting of a high-resolution camera, external illumination and a motorised sample holder, is connected via USB to a smartphone or another mobile device (laptop, tablet). An application on this device controls the external camera and transmits the captured images encrypted to a cloud platform. There the image analysis and recognition are carried out, the results are transferred back to the smartphone or to a doctor/health centre. 
in image recognition compared to other algorithmic models (LeCun et al., 2015).

\section{Conclusions}

At the present state, a fast and simple preparation and microscopy of blood samples with the recent system can be achieved. Nevertheless, the current magnification of 400x is sub-optimal for the analysis of small blood components (thrombocytes, parasites). Also for the reliability of diagnostic results, many images of one sample are essential. Therefore further development of the system is imperative. Furthermore, it can be said that nearly every application in medicine/diagnostics, environmental and food technology is imaginable, where mobile microscopy and automated analysis is advantageous. This opportunity will also enable many researchers to investigate biological samples of their area in a more efficient way.

\section{Key Points}

- A mobile microscope for the examination of blood samples is beneficial for several

- Applications: cell counting and differentiation, identification/ quantification of parasites

- It enables a fast and reliable diagnosis of blood-related diseases

- Automated image analysis is on the basis of neural networks and deep learning

- Experts on site and laboratories are not needed

- Utilisation as a POCD tool in remote-rural areas and endemic regions is a goal

\section{Acknowledgements}

The authors thank Tobias Hagemann for his efforts to investigate the feasibility of malaria detection in Africa. Work for this manuscript was financed by the Ministry of Science, Research and Culture of the federal state of Brandenburg, Germany in the HealthCampus initiative "Digital and analogue companions for an ageing population (DigiLog)" under grant no. GeCa: H22805/002/004.

\section{References}

1. Benoist BD, McLean E, Egll I, Cogswell M (2008) World Health Organization. Worldwide prevalence of anaemia 1993-2005: WHO global database on anaemia (2008). Edited by Bruno de Benoist, Erin McLean, Ines Egli and Mary Cogswell. ISBN: 978 9241596657

2. LeCun Y, Bengio Y, Hinton G (2015) Deep learning. Nature 521:436-444 http://dx.doi.org/10.1038/nature14539

3. Malaria Atlas Project: http://www.map.ox.ac.uk/. Version: 2017

4. Schulze K, Tillich UM, Dandekar T, Frohme M (2013) PlanktoVision- an automated analysis system for the identification of phytoplankton. BMC Bioinformatics 2013 14:115. http:/! dx.doi.org/10.1186/1471-2105-14-115

5. White C, Zainasheff J (2010) Yeast: the practical guide to beer fermentation. Brewers Publications. ISBN-13: 978-0937381960 